Gynäkologische Endokrinologie 2020 18:187-188 https://doi.org/10.1007/s10304-020-00340-z

(c) Springer Medizin Verlag $\mathrm{GmbH}$, ein Teil von Springer Nature 2020

\section{B. Sonntag' $\cdot$ G. Griesinger ${ }^{2,3} \cdot$ R. Felberbaum ${ }^{4}$}

'Facharztzentrum für Kinderwunsch, Pränatale Medizin, Endokrinologie und Osteologie, amedes experts Hamburg, Hamburg, Deutschland

${ }^{2}$ Sektion für Gynäkologische Endokrinologie und Reproduktionsmedizin, Universitätsklinikum SchleswigHolstein, Campus Lübeck, Lübeck, Deutschland

${ }^{3}$ Universitäres Kinderwunschzentrum Lübeck und Manhagen, Universitätsklinikum Schleswig-Holstein, Lübeck, Deutschland

${ }^{4}$ Centre of Reproductive Medicine, Klinikverbund Kempten-Oberallgäu, Kempten (Allgäu), Deutschland

\title{
Gesundheit der Kinder nach assistierter Reproduktion
}

Nach einer In vitro-Fertilisation (IVF) mit oder ohne intrazytoplasmatische Spermieninjektion (ICSI) werden allein in Deutschland jährlich ca. 13.000 Kinder geboren. Die assistierte Reproduktion ist mit einer jährlichen Durchführung von mittlerweile über 100.000 Behandlungszyklen einschließlich der nach Einfriertechniken resultierenden Auftauzyklen hier somit nicht mehr als Randgebiet der Medizin anzusehen. Das medizinische Vorgehen von der Indikationsstellung über die Beratung der Paare bis hin zur eigentlichen ovariellen Stimulationstherapie und Eizellgewinnung erfolgt hochspezialisiert in aktuell 139 Kinderwunschzentren in Deutschland, welche ihre durchgeführten Behandlungen seit vielen Jahrzehnten im Rahmen einer freiwilligen Selbstverpflichtung im Deutschen IVF-Register (DIR) dokumentieren. Insbesondere über die notwendige Schnittstelle des ärztlich-medizinischen Bereichs zu den biologischen Spezialisten in den IVFLaboren der Kinderwunschzentren ist das Fach im besonderen Maße durch Interdisziplinarität gekennzeichnet. Gerade die letzten Jahre waren geprägt von biologisch-technischen Veränderungen bei den Kulturverfahren der Embryonen (z. B. Blastozystenkultur), aber auch bei den Einfrierverfahren (zunehmende Umstellung auf Vitrifikation). Diese Innovationen fließen auch ein in eine zunehmend individuellere Beratung der Paare mit dem Ziel einer Verbesserung der Erfolgsraten bei gleichzeitig maximaler Sicherheit für die Gesundheit der Patientin. In kaum einem anderen Gebiet der Medizin wird aber durch das aktuelle Handeln auch bereits die gesundheitliche Basis der nachfolgenden Generation mitbeeinflusst. Dies dürfte nicht nur an den eingesetzten medizinischen Therapien und Technologien im IVF-Labor liegen, sondern teilweise auch durch die Elterngeneration mit ihrer zugrunde liegenden und mitunter multifaktoriellen Subfertilität mitbedingt sein. Es ist unsere Verantwortung, als durchführende Ärzte auch diesen Aspekt durch die Auswertung vorliegender Daten bei der risikoadaptierten Therapieauswahl in unsere Beratung zu integrieren. Im vorliegenden Heft wird daher explizit auf Daten zur Gesundheit der Kinder nach assistierter Reproduktion beginnend in der vorgeburtlichen Periode und bis zum mittlerweile jungen Erwachsenalter eingegangen. Von Versen-Höynck beschäftigt sich in ihrem Beitrag detailliert mit den vom Konzeptionsmodus abhängigen perinatalen und peripartalen Risiken. Baston-Büst stellt die biologische Sicht zum Einfluss beispielsweise von Kulturmedien und Gefriertechniken insbesondere vor dem Hintergrund vermuteter kardiovaskulärer Risiken der Nachkommen dar. Sonntag et al. und Ludwig et al. stellen in zwei Beiträgen Daten aus der Deutschen ICSI-Follow-up-Studie zur Pubertätsentwicklung bzw. den kardiovaskulären und metabolischen Risiken der Jugendlichen nach assistierter Reproduktion unter Beachtung der maternalen Risikofaktoren vor. Sitzberger et al. konzentrieren sich 
in einer systematischen Übersicht auf das Vorliegen kardiovaskulärer Fehlbildungen, Sutcliffe et al. auf Studien zum Krebsrisiko der Kinder.

Nicht zuletzt stellt es eine ganz wesentliche Aufgabe dar, diese vorhandenen Kenntnisse aus einem hochspezialisierten Bereich der Medizin für die Betreuung der Schwangeren und Kinder durch unsere KollegInnen in den entsprechenden Disziplinen aufzubereiten und zur Verfügung zu stellen.

Wir wünschen Ihnen viel Freude und neue Erkenntnisse bei der Lektüre!

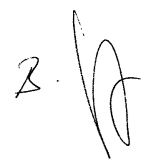

B. Sonntag

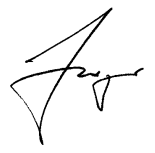

G. Griesinger

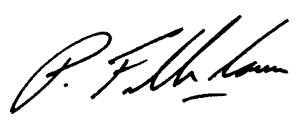

R. Felberbaum

\section{Korrespondenzadresse}

Prof. Dr. med. B. Sonntag

Facharztzentrum für Kinderwunsch, Pränatale Medizin, Endokrinologie und Osteologie, amedes experts Hamburg Mönckebergstr. 10, 20095 Hamburg, Deutschland

Barbara.Sonntag@amedes-group.com

Interessenkonflikt. B. Sonntag, G. Griesinger und R. Felberbaum geben an, dass kein Interessenkonflikt besteht.

\section{Aktuelle Buchempfehlungen aus dem Springer-Verlag}

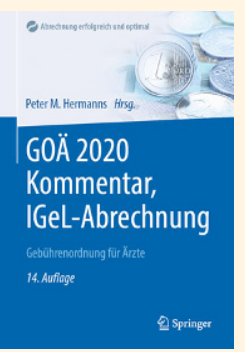

\section{GOÄ 2020 Kommentar, IGeL-Abrechnung}

Gebührenordnung für Ärzte

Hermanns, Peter M. (Hrsg.)

XXIV, 896 Seiten

2020| 14. Auflage

Springer-Verlag

978-3-662-60547-9 (ISBN)

$79,99 €$

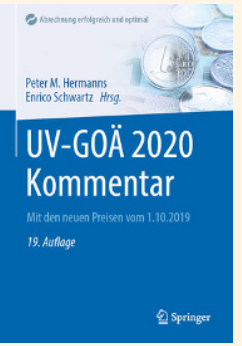

\section{UV-GOÄ 2020 Kommentar}

Mit den neuen Preisen vom 1.10.2019

Hermanns, Peter M., Schwartz, Enrico (Hrsg.)

XXIII, 767 Seiten

2020| 19. Auflage

Springer-Verlag

978-3-662-60549-3 (ISBN)

$69,99 €$

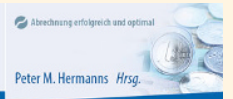

\section{EBM 2020 Kommentar Kinderheilkunde}

Kompakt: Mit Punktangaben, Eurobeträgen, Ausschlüssen, GOÄ

EBM 2020

Kommentar

Kinderheilkunde

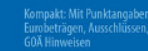

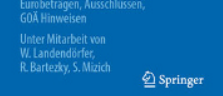

Hinweisen

Hermanns, Peter M. (Hrsg.)

XX, 429 Seiten

2020

Springer-Verlag

978-3-662-61465-5 (ISBN)

$39,99 €$

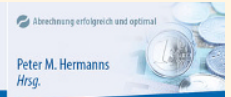

EBM 2020

Kommentar

Allgemeinmedizin

Kompakt: Mit Punktangaben
Eurobetiogen, Ausschlisssen

Gon Hinweisen
Unter Mitabeit von
I. Buther

פSpringer

\section{EBM 2020 Kommentar Allgemeinmedizin}

Kompakt: Mit Punktangaben, Eurobeträgen, Ausschlüssen, GOÄ Hinweisen

Hermanns, Peter M. (Hrsg.)

$\mathrm{XX}, 411$ Seiten

$2020 \mid$ 1. Aufl. 2020

Springer-Verlag

978-3-662-61501-0 (ISBN)

$39,99 €$

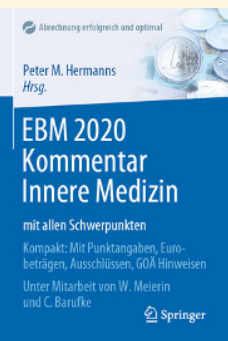

EBM 2020 Kommentar Innere Medizin mit allen Schwer-

\section{punkten}

Kompakt: Mit Punktangaben, Eurobeträgen, Ausschlüssen, GOÄ

Hinweisen

Hermanns, Peter M. (Hrsg.)

XXII, 685 Seiten

$2020 \mid$ 1. Aufl. 2020

Springer-Verlag

978-3-662-61503-4 (ISBN)

$49,99 €$ 